\title{
Understanding Recent Insights in Sleep and Posttraumatic Stress Disorder from a Research Domain Criteria (RDoC) Framework
}

\author{
Monica R. Kelly ${ }^{1}$ - William D. S. Killgore ${ }^{1,2}$ • Patricia L. Haynes ${ }^{1,2,3}$
}

Published online: 13 October 2016

(C) Springer International Publishing AG 2016

\begin{abstract}
Posttraumatic stress disorder (PTSD) is associated with sleep disturbances, including insomnia, nightmares, sleep-disordered breathing, and REM abnormalities. Recent studies have expanded our knowledge of the neurobiology of trauma and sleep. In addition, intervention research has provided valuable information about how sleep treatments affect PTSD symptoms and how PTSD treatments affect sleep symptoms. This review of recent literature aims to move away from the categorical approach that currently dominates our conceptualization of trauma response and sleep disorders. Possible mechanisms of both trauma and sleep disturbance are posed within the framework put forth by the National Institute of Mental Health's (NIMH) Research Domain Criteria (RDoC) project. By examining domains of dysfunction that cut across disorders, $\mathrm{RDoC}$ may guide future research and treatment of the maladaptive neurobiological and behavioral correlates of both trauma and sleep disturbances.
\end{abstract}

Keywords Posttraumatic stress disorder · Insomnia ·

Nightmares $\cdot$ Cognitive behavioral therapy $\cdot$ Obstructive sleep apnea

This article is part of the Topical Collection on Sleep and Psychological Disorders

Patricia L. Haynes

thaynes@email.arizona.edu

1 Department of Psychology, University of Arizona, 1503 E. University Blvd, Tucson, AZ 85721, USA

2 Department of Psychiatry, University of Arizona, 1501 N. Campbell Ave, Tucson, AZ 85724-5002, USA

3 Department of Health Promotion Sciences, University of Arizona, 1295 N. Martin Ave, P.O. Box 245209, Tucson, AZ 85724-5209, USA

\section{Introduction}

The DSM-5 diagnostic criteria for posttraumatic stress disorder (PTSD) consists of several specific daytime and nighttime emotional, cognitive, and behavioral symptoms [1], many of which involve or may be affected by sleep-related phenomena. Sleep difficulties, in particular, have long been considered a hallmark of the disorder [2], with upward of $90 \%$ of patients reporting some form of sleep disturbance [3-5]. Within the past few years, two new diagnostic categories regarding trauma-related sleep disturbances were proposed, including trauma-induced insomnia [6], which may also be a prodrome of chronic insomnia and PTSD, and trauma-associated sleep disorder [7], which encompasses a variety of symptoms across multiple parasomnias. While categorical approaches to understanding the relationship between sleep and trauma may be helpful for treatment communication and billing purposes, these conceptualizations have been limited in their ability to further scientific understanding of the trauma response and to facilitate the development of effective interventions [8]. Therefore, research that focuses more directly on understanding symptoms in terms of systemic malfunctions and interactions is likely to inform new treatment approaches and improve overall outcomes.

In an effort to advance the field beyond the limitations of the current categorical nosology, the National Institute of Mental Health (NIMH) established the Research Domain Criteria (RDoC) [9]. The RDoC approach critiques the current psychiatric diagnostic system for clustering non-distinct, overlapping symptoms without a unifying theoretical or neurobiological rationale. The $\mathrm{RDoC}$ project promotes research investigating neuroscience-based dimensions of psychiatric dysfunction as an alternative to studying categorical syndromes. These dimensions, or constructs within domains of functioning (see Table 1), map onto transdiagnostic behavioral 
Table 1 NIMH RDoC domains and constructs [9]

\begin{tabular}{|c|c|}
\hline Domains & Constructs \\
\hline \multicolumn{2}{|c|}{ Negative valence systems } \\
\hline & Active threat ("fear") \\
\hline & Potential threat "anxiety") \\
\hline & Sustained threat \\
\hline & Loss \\
\hline & Frustrative nonreward \\
\hline \multicolumn{2}{|c|}{ Positive valence systems } \\
\hline & Approach motivation \\
\hline & Initial responsiveness to reward \\
\hline & Sustained responsiveness to reward \\
\hline & Reward learning \\
\hline & Habit \\
\hline \multicolumn{2}{|c|}{ Cognitive systems } \\
\hline & Attention \\
\hline & Perception \\
\hline & Working memory \\
\hline & Declarative memory \\
\hline & Language behavior \\
\hline & Cognitive control \\
\hline \multicolumn{2}{|c|}{ Social processes } \\
\hline & Affiliation and attachment \\
\hline & Social communication \\
\hline & Perception and understanding of self \\
\hline & Perception and understanding of others \\
\hline \multicolumn{2}{|c|}{ Arousal/regulatory systems } \\
\hline & Arousal \\
\hline & Circadian rhythms \\
\hline & Sleep-wakefulness \\
\hline
\end{tabular}

symptoms. Each construct can be assessed across various units of measurement including genes, molecules, cells, circuits, physiology, and self-report. RDoC proposes that successful treatment outcomes must begin with understanding and utilizing basic domains and constructs to develop a precision medicine-style approach to mental health problems [10].

According to the RDoC, trauma-related symptoms of PTSD fit primarily within the sustained threat construct of the negative valence system, whereas sleep/wakefulness is primarily classified as a construct within the arousal and regulatory systems domain. However, even within RDoC definitions, sleep is inconsistently identified. Sleep-related behaviors are present in the loss construct under negative valence systems, but they are absent from biological drives (i.e., appetite, libido) in the construct of sustained threat within the same domain. Additionally, interactions are possible between various domains, as appears to be the case in arousal and negative valence in the context of sleep and trauma exposure [11].
Supporting the notion of inter-domain interactions, sleep problems are strongly linked to the maladaptive emotional and cognitive processing symptoms associated with PTSD [11]. Furthermore, recent research has demonstrated that sleep problems may play an etiological role in the development of PTSD [12, 13]. By shifting the focus of research efforts towards the mechanisms of sleep and PTSD symptomatology, we may better understand the underlying constructs, as well as inform innovative and ideographically targeted treatments [14].

This review aims to provide an alternative structure for examining dysfunctional domains related to trauma and sleep. Below, we review articles published during the preceding 3 years addressing sleep and PTSD within the context of the RDoC framework. First, we discuss new findings across RDoC units of measurement. Next, we outline recent progress in treatment approaches for trauma and sleep symptoms as they apply to these dysfunctions. Finally, we conclude with a commentary on future directions for research in $\mathrm{RDoC}$ domains in addition to treatments relevant to trauma exposure and sleep. By highlighting the newest developments, we hope to promote a better understanding of what is known about sleep and trauma exposure regarding etiology, interactions, maintenance, and resolution of dysfunctions within and across the basic domains.

\section{RDoC Framework-Based Correlates of Trauma and Sleep}

Genes A number of studies have explored the genetic correlates of sleep as they relate to trauma exposure. One such study assessing PTSD risk in urban-dwelling, traumaexposed African Americans identified a female-specific genetic polymorphism (adenylate cyclase-activating polypeptide 1 receptor type 1; ADCYAP1R1). This gene encodes the receptor for pituitary adenylate cyclase-activating polypeptide (PACAP) [15], which is believed to modulate sleep/wake and promote rapid eye movement (REM) sleep [16]. Recently, an ADCYAP1R1 replication study demonstrated that a genotype by trauma severity interaction, rather than genotype alone, resulted in an increased risk of PTSD in females [17]. The same group found ADCYAP1R1 is associated with amygdala hyperactivity and reduced amygdala-hippocampus connectivity [18•]. These data have implications of genetic mechanisms related to increased risk of dysfunction in arousal, negative valence, and cognition in terms of emotional reactivity, attentional bias to threat, avoidance, and memory retrieval. However, the focus on genetics is relatively new, and more research is needed to determine genetic profiles and other factors resulting in increased risk of developing these dysfunctions in other populations. 
Molecules Neurotransmitter imbalances likely contribute to both PTSD and sleep difficulties. Levels of the neurotransmitter gamma-aminobutyric acid (GABA), known for its inhibitory function, are lower in the right anterior insula of individuals diagnosed with PTSD relative to controls and individuals with elevated self-reported state and trait anxiety [19]. Lower GABA was also shown in the parieto-occipital cortex (POC) among individuals with PTSD, and the association between PTSD and POC GABA was partially mediated by selfreported insomnia severity [20]. These data suggest that individuals with PTSD may benefit from GABA-enhancing medications that target general arousal and insomnia symptoms.

Cells A number of studies have shown that reduced gray matter volume in regions of the ventromedial prefrontal cortex (vmPFC) is associated with sleep complaints such as chronic insomnia [21], excessive daytime sleepiness [22], and obstructive sleep apnea [23]. Structural changes in the brain are also present in individuals with both sleep and trauma-related symptom complaints. Insomnia and nightmares were associated with reduced gray matter volume in the amygdala, hippocampus, anterior cingulate cortex, and insula in traumaexposed Stockholm transportation workers who developed PTSD relative to workers who did not develop PTSD [24]. Reduced frontal lobe volume was associated with worse subjective sleep quality in combat-exposed Gulf War Veterans, regardless of PTSD diagnosis [25]. Many previous studies have demonstrated reduced hippocampal volume in PTSD, especially in severe cases [26]. However, recent findings suggest that difficulties with sleep may substantially account for the relationship between PTSD and hippocampal volume [27]. Sleep difficulties, especially insomnia, may play a greater role than trauma-related symptoms in structural changes; however, causality and implications for the interplay between arousal, negative valence, and cognitive systems remain unclear.

Circuits In 2006, Rauch and colleagues proposed the neurocircuitry model of PTSD, theorizing a series of limbic system dysfunctions, paired with fear conditioning, as the diathesis-stress interaction necessary for PTSD symptom initiation [28]. The model suggests the onset of PTSD symptoms results from (a) overreactivity to threat-relevant stimuli in the amygdala with (b) failure of the ventromedial prefrontal cortex (vmPFC) to exert adequate top-down suppressive control over both the amygdala and hippocampus. Representing a variety of $\mathrm{RDoC}$ domains, this process of limbic hyperactivity and insufficient top-down regulation is hypothesized to result in hyperarousal (arousal and regulatory systems), difficulties related to fear extinction and differentiation between actual versus imagined threat (negative valence systems), as well as elevated emotionality and insufficient attention/response suppression in relation to trauma memories (cognitive systems)
[28]. This model has received considerable support from functional neuroimaging studies of PTSD [29, 30].

The circuit-level dysfunction observed in PTSD during wake is strikingly similar to deficient neurological regulatory functioning and related increases in psychopathological symptomatology following sleep deprivation in healthy individuals. Past findings demonstrate one night of total sleep deprivation, relative to a night of sleep, produces increased amygdala reactivity to negatively valenced visual stimuli [31]. Additionally, sleep deprivation leads to weaker functional connectivity between the medial prefrontal cortex (mPFC) and the amygdala, but it is associated with stronger connectivity between the amygdala and sleep/wake centers of the brainstem (locus coeruleus, midbrain), suggesting greater autonomic activation [31]. Recent studies have built upon these findings to show that even self-reported lack of sleep is associated with altered functional connectivity between the medial prefrontal control regions and the emotionally responsive amygdala, suggesting deficient capacity to regulate emotions when sleep is reduced [32]. As functional connectivity is altered, there is a significant increase in symptoms of psychopathology such as anxiety and depression, and a reduction in self-perceived emotional capacities [32], all findings that have been previously linked to sleep deprivation [33, 34]. These data in non-trauma-exposed individuals suggest a mechanistic pathway by which symptoms traditionally thought of as trauma-related may be moderated by sleep disturbance. Specifically, lack of sleep may weaken the ability of topdown regulation of limbic emotion response systems by the prefrontal cortex, a process that closely parallels the neurocircuitry model of PTSD.

Physiology In addition to overall sleep loss, sleep fragmentation (e.g., frequent, mid-sleep arousals) plays an important role in PTSD symptom progression. Despite inconsistencies across studies, a meta-analysis comparing sleep in individuals with and without PTSD found greater stage 1 sleep and reduced slow wave sleep in connection with increased REM density. These results indicate frequent, brief mid-sleep awakenings and REM sleep abnormalities in individuals with PTSD [35]. As described by Ross [2] and later expanded on by Mellman [36], disturbances of REM are especially relevant for PTSD. REM sleep is strongly associated with memory consolidation and affective processing, as well as nightmares $[37,38]$, as it provides the opportunity for affective reactivity attenuation while preserving memory content [39]. Recent studies have documented an association between PTSD and increased localized glucose metabolism in the brainstem, limbic system, and striatum during both wake and REM sleep [38]. Increased glucose metabolism in these regions maps onto behavioral symptoms of PTSD: intrusion (fear reactions, failure to habituate), hyperarousal (sleep/wake disturbance), 
and avoidance (impaired reward processing) [38]. Additionally, individuals with PTSD demonstrate stronger discrepancies in hypermetabolism throughout limbic and paralimbic regions in REM sleep versus wake as compared to individuals with major depressive disorder (MDD), which may contribute to the prevalence of nightmares in PTSD but not in MDD [40 ${ }^{\bullet}$.

Recent findings build upon Walker and Van der Helm's theory that REM sleep promotes adaptive emotional memory consolidation [41]. A cross-sectional study of non-treatmentseeking, trauma-exposed, and urban-dwelling African Americans between ages 18 and 35 found disrupted and reduced REM sleep for individuals with more proximate trauma exposure and greater PTSD-related symptoms. However, REM sleep architecture appeared non-disrupted in individuals with chronic PTSD [42]. These findings are consistent with previous literature in treatment-seeking, non-minority [43], and military populations [44]. Trauma-exposed individuals without PTSD, compared to those with PTSD, exhibit elevated right frontal theta power (4-8 Hz) during REM sleep. This physiologic marker is indicative of wakeful encoding and sleep-dependent memory consolidation, while frontal asymmetry is associated with affective processing [45]. Thus, new data continue to support the hypothesis that psychophysiological correlates representative of emotional memory processing are present in REM sleep, and promotion or consolidation of REM sleep may be a protective factor in regard to traumarelated symptoms.

Breathing is another autonomic function that is an important physiological variable in the $\mathrm{RDoC}$ arousal construct. Sleep fragmentation, even more so than sleep deprivation, results in upper airway resistance in healthy individuals. This may result from a combination of greater attenuation of upper airway dilator musculature and repeated instances of endorphin release generated during frequent mid-sleep arousals [46]. Therefore, frequent awakenings in individuals with PTSD may put these individuals at a greater risk of developing sleep-disordered breathing (SDB) [47]. Comorbidity of PTSD and SDB has a high prevalence, implications for worse symptomatology, and an altered clinical presentation. One recent study found that obstructive sleep apnea (OSA) was present in more than half of individuals seeking treatment for PTSD [48]. This comorbidity, as compared to either disorder alone, was related to worse daytime symptoms and reduced quality of life [48]. Additionally, greater percent of time in REM sleep was positively associated with nightmares in individuals with PTSD and untreated OSA [49••], further supporting a link between REM sleep and endogenous cues for sustained threat. Populations that do not present with typical indicators of OSA, such as older age or higher body mass index (BMI) [50], may be overlooked during routine screening for sleep-disordered breathing. The rate of OSA among active duty combat Veterans with sleep problems is as high as
$78 \%$ [51]. One recent study found that $69 \%$ of young military Veterans with sub-threshold BMI, seeking treatment of PTSD, scored as high risk for OSA [52]. These data highlight the need for screening of sleep-disordered breathing symptoms in individuals presenting with PTSD symptoms even in the absence of traditional signs of apnea.

Taken together, these data suggest that adequate sleep, especially REM sleep, is indicated in the promotion of affect and arousal modulation as well as emotional memory processing [53]. Interventions targeting arousal reduction may help moderate limbic hyperactivity to promote sleep, while sleep/wake pattern and sleep-disordered breathing stabilization are likely to enhance fear extinction and habituation.

Self-Report More than three decades of research show that PTSD is associated with major complaints of insomnia and nightmares [54-56]; however, subjective experience of shortened total sleep time and extended sleep onset latency is incongruent with objectively assessed sleep [57]. Recent research has investigated the relationship between selfreported sleep and trauma-related symptoms to inform diagnosis and treatment planning. In Vietnam-era veterans, a recent study found that nightmare-related PTSD symptom severity and distress were associated with subjective sleep fragmentation, reduced total sleep time, and increased delay in sleep onset. Longer subjective sleep onset latency (SOL) was observed on nights with, versus without, nightmares and was associated with bedtime ratings of daytime stressor frequency [58]. These findings suggest that the successful treatment of PTSD-associated nightmares may involve improving SOL and implementing coping skills for managing daytime stressors.

Current research studies suggest a bidirectional relationship between self-reported sleep and symptoms of PTSD. One study found that specific trauma types (e.g., sexual trauma, physical assault, and witnessing sudden violent death) are linked with subsequent sleep disturbance severity [59]. Also, sleep disturbance may initiate or exacerbate PTSD. Several recent self-report studies suggested that insomnia increases the likelihood of PTSD development following trauma [60, 61]. Subjective difficulty falling and staying asleep prior to deployment predicts later development of PTSD following combat exposure [12]. Both subjective and clinician-rated sleep disturbances and associated daytime difficulties predict PTSD and other internalizing disorders in National Guard troops [13]. Sleep may be best conceptualized as moderating and/or mediating the relationship between trauma exposure and PTSD. However, future studies are needed to replicate and expand on these data that employ prospective measures of sleep, such as daily sleep diary.

Given the complexity of the clinical presentation of individuals with comorbid sleep disorders and PTSD, researchers are conducting exploratory analyses to identify symptom 
patterns for hypothesis generation. A recent study employed exploratory cluster analysis of symptoms in military veterans with trauma and sleep-related difficulties. The authors identified three distinct symptom profiles related to sleep, presence or absence of parasomnia symptoms, and a continuum of daytime PTSD symptom severity. All three profiles included poor sleep quality [62]. These preliminary data support viewing sleep and trauma-related symptoms on a spectrum and evaluating treatment effects as a function of symptom profiles rather than diagnoses.

\section{Recent Treatment Studies}

Treatment for Nightmares in PTSD Nightmares are among the most common parasomnias experienced by individuals with PTSD [63]. Nightmare-focused psychotherapy aims to reduce reactivity to internal stimuli and response to sustained threat. Imagery rehearsal therapy (IRT) is a psychotherapy for nightmares that involves writing an altered version of a trauma-related nightmare and rehearsing the changed narrative [64]. Findings are mixed about the efficacy of IRT [65, 66]. A recent meta-analysis demonstrated that IRT as compared to prazosin, an alpha-aderenergic blocker previously shown to be effective in reducing nightmares [64], produced equivalent reductions in nightmare frequency and associated sleep and trauma symptoms [67]. As such, the American Academy of Sleep Medicine and Veterans Administration/ Department of Defense endorses prazosin for the treatment of PTSD nightmares $[64,68]$ which was further supported by two prazosin meta-analyses published in $2015[69,70]$.

While prazosin is often associated with positive outcomes in PTSD, there are a number of limitations to its effectiveness. In a recent study, prazosin produced clinically significant reductions in nightmares, as well as modest reductions in PTSD symptoms and improvements in sleep quality for active-duty military personnel [71]. However, these positive findings do not hold for veterans with PTSD and co-occurring substance dependence. The effects of prazosin may be negated by the impact of heavy alcohol consumption on sleep architecture, specifically in regard to producing REM rebound [72]. It should be noted that prazosin does not ultimately resolve the underlying issue and discontinuation may result in a rebound of nightmares [73]. Residual PTSD symptoms following treatment with prazosin suggest exploring its use in conjunction with other treatments to counter general arousal and negative valence dysfunctions [71]. Despite tolerability and symptom improvement, emerging data suggest that much work remains to fully elucidate the utility and safety for use of prazosin in PTSD and sleep disturbances, especially in individuals with other psychiatric comorbidities and non-military populations.
Research is beginning to examine whether nightmarefocused treatments administered concurrently with PTSD treatments produce an improved response. Previous work has demonstrated effectiveness of combined nightmare rescripting and emotional processing for both nightmare frequency and PTSD symptom severity in both civilian [74] and veteran populations [75]. Exposure, relaxation, and rescripting therapy (ERRT) incorporates IRT techniques with sleep and trauma-related psychoeducation, relaxation training and problem-focused coping skills, and exposure (focusing on trauma-related nightmares, identifying themes, and verbal rehearsal in session). Results of a recent meta-analysis suggest that psychotherapy for nightmares (e.g., IRT and ERRT) produces large effect sizes for reductions in nightmare frequency and PTSD symptoms at the end of treatment and during follow-up. However, it appears that greater duration of emotional processing during treatment, with or without exposure, produced equivalent or superior effect sizes in terms of reduction in nightmare frequency as compared to exposure alone. These data suggest cognitive techniques to reduce thought suppression and improve affect regulation ability may be at least as important as habituation for treatment of nightmares [76].

Comorbid insomnia may further complicate treatment of individuals with trauma-related nightmares. Consistent with previous work in non-veteran populations, ERRT modified for military populations (ERRT-M) demonstrated reduced nightmare frequency and self-reported insomnia severity at 2 months posttreatment [77]. However, veterans who did not experience a clinically significant improvement entered the study with greater insomnia severity [77]. A recent metaanalysis of nightmare treatments showed sleep and trauma symptom improvement was substantially bolstered by combining cognitive behavioral therapy for insomnia (CBT-I) with IRT [67]. Therefore, directly addressing sleep quality and quantity either prior to or in conjunction with nightmare treatment may produce the greatest benefit for trauma-exposed individuals with both insomnia symptoms and nightmares.

Treatment for Insomnia in PTSD Cognitive behavioral therapy for insomnia (CBT-I), the gold-standard treatment for insomnia, consists of multiple treatment components mapping onto behaviors and cognitions that primarily fall under RDoC arousal and regulatory systems domains. Stimulus control reduces the pairing of arousing behavioral cues with the bed, while increasing associations with sleep. Sleep restriction limits the average time spent in bed to build homeostatic sleep drive. Sleep hygiene decreases sleep-hindering and increases sleep-promoting health behaviors. Cognitive therapy involves challenging anxious arousal and maladaptive sleep-related beliefs. In a community-based sample diagnosed with comorbid PTSD and insomnia, CBT-I versus waitlist control produced gains in self-reported sleep quality, parasomnia reduction, and general functioning. CBT-I also resulted in increased 
subjectively and objectively assessed total sleep time, and all gains were sustained at 6 months post-intervention [78•]. Another meta-analysis of CBT with a sleep focus that included CBT-I, as well as IRT and ERRT, found medium to large effects for improved subjective insomnia and PTSD symptoms and small to medium effects for depression [79]. Successful CBT-I treatment in active-duty military personnel with PTSD was also associated with decreased inflammatory gene expression and depression severity [80], supporting previous work indicating inflammatory response as an important physiological mechanism in both arousal and negative valence systems [81]. Overall, recent data suggest that sleep treatment, and CBT-I specifically, improves sleep as well as PTSD and depression with maintenance of improvements over time.

\section{New Treatments for Both Insomnia and Nightmares in} PTSD Previous studies have evaluated group modalities for individuals with PTSD experiencing insomnia and/or nightmares with positive results for both sleep and trauma-related symptoms [82, 83]. More recently, Haynes and colleagues developed and tested Cognitive Behavioral Social Rhythm Therapy (CBSRT) in veterans with moderate to severe comorbid insomnia, MDD, and PTSD [84]. CBSRT is a non-traumafocused, chronobiologically informed psychotherapy based on the social rhythm model of mood disruption [85] that incorporates elements of both IRT and CBT-I [84]. CBSRT resulted in a relatively low attrition rate $(13 \%)$, and a substantial proportion of participants achieved subclinical levels of SOL (88\%) and wake after sleep onset (WASO; $75 \%$ ), as well as reduced PTSD avoidance symptoms [84].

Another recent study included three sessions of standardized sleep-oriented psychoeducation and self-hypnosis (audio-recorded progressive muscle relaxation, guided imagery, and affirmations) as an adjunct to cognitive processing therapy (CPT) for PTSD in female assault victims with significant sleep disturbance [86]. As compared to daily monitoring of sleep and trauma-related symptoms alone, sleeporiented self-hypnosis produced large effect sizes for SOL (without full remission of insomnia) and significant decreases in depression symptoms [86]. Dismantling studies of these multicomponent psychotherapies may help to identify mechanisms of change and critical aspects of sleep treatment in complex cases of trauma exposure and sleep disturbance comorbidity.

Sleep Changes with PTSD Treatments Selective serotonin reuptake inhibitors (SSRIs) are effective for the treatment of PTSD but not as effective as psychotherapy [87]. Like psychotherapy, treatment by SSRIs is associated with improved sleep quality [68]. Unfortunately, these medications also have sleep-related side effects, including insomnia and periodic limb movement sleep disorder [68]. Of concern, greater usage of hypnotics is associated with less sleep improvement over the course of CBT for PTSD [88]. Therefore, these medications should be used with caution when treating individuals with insomnia symptoms and PTSD.

Cognitive behavioral therapies (CBT) for PTSD include techniques such as fear memory exposure and emotional processing to address RDoC dysfunctions of negative valence and arousal systems. Sleep loss and fragmentation are known to alter affective reactivity and cognitive functioning; thus, symptoms of insomnia and nightmares may impede or blunt the effects of CBT for PTSD [11]. Studies are necessary to test this theory specifically; however, a few have attempted to assess the influence of sleep and related symptoms on PTSD treatment outcomes. Preliminary case comparative findings examined factor combinations in relation to positive response to CBT for PTSD. Results showed that absence of severe SOL at pre-treatment was the most representative factor related to successful CBT for PTSD across all combinations [89]. Another study examined Ehler and Clark's trauma-focused cognitive therapy for PTSD (CT-PTSD) in a London community-based sample. Individuals with lower baseline self-reported sleep quality and depression had slower improvement in PTSD symptoms as compared to nondepressed individuals with better baseline sleep quality [90]. Taken together, research suggests that treatment of prolonged SOL and poor sleep quality prior to CBT for PTSD may improve outcomes for a significant subset of individuals with PTSD.

Intervention researchers have previously found insomnia symptoms to be one of the most frequent and severe residual symptoms following CBT for PTSD, possibly due to the lack of hyperarousal-specific techniques [88, 91]. A recent study of CBT for PTSD response demonstrated sustained improvement in sleep symptoms assessed by the Pittsburgh Sleep Quality Index and sleep items on the Clinician-Administered PTSD Scale. However, individuals never fully achieved remission of sleep disturbance [92]. One preliminary study using daily sleep diaries found negligible changes on all sleep indices in CBT for PTSD responders, $85 \%$ of whom met insomnia disorder criteria at pre-treatment [93]. A small study examined the effect of Eye Movement Desensitization and Reprocessing (EMDR) therapy on objectively measured sleep in Brazilian individuals with assault or kidnapping-related PTSD. The authors found a pre-to-post treatment reduction in WASO assessed by polysomnography, but not for other arousal-relevant variables such as SOL [94]. Overall, recent data continues support the notion that CBT for PTSD does not adequately treat sleep symptoms, and insomnia treatment may prove beneficial in conjunction with PTSD psychotherapy [88].

CPAP for Obstructive Sleep Apnea Prevalence estimates of PTSD comorbid with sleep disordered breathing range from 
$57 \%$ for active-duty military personnel [48] to $90 \%$ for civilian female sexual assault victims [95]. These rates are especially large in the Veteran's Administration (VA) system given the high prevalence of middle-age and older men [96]. Continuous positive airway pressure (CPAP) therapy is the gold standard treatment for obstructive sleep apnea (OSA). In comorbid PTSD and OSA, objectively assessed CPAP compliance produces substantial benefits regarding improved daytime functioning and reduction of PTSD symptoms including trauma-related nightmares [49••]. Unfortunately, blunted response to CPAP therapy was also observed in comorbid PTSD and OSA, relative to individuals with only OSA [48]. Poor adherence to CPAP treatment is common in comorbid PTSD and OSA and has recently been found to be especially prevalent in ethnic minorities with both PTSD and OSA [49・•]. Given the positive impact of successful CPAP therapy on mental health and general functioning, screening and treatment of OSA should be considered a priority for individuals with PTSD. Future studies are needed to establish best practices for promoting adherence in comorbid PTSD and OSA and particularly for ethnic minorities.

\section{Conclusions and Future Directions}

In recent years, important advances have broadened our understanding of the complex interplay between sleep and trauma, especially in the realms of neurobiology and treatment. Sleep difficulties, particularly dysfunctions of REM sleep, play a significant role in trauma-related symptom development and resiliency. Despite this important work, there is still much we do not know about these conditions, particularly in regard to how constructs of arousal and sustained threat overlap. Employment of cutting-edge neuroimaging techniques may help illuminate causation regarding changes to functional or structural neural integrity as a function of trauma, sleep, and treatment. Case comparative and mixed methods research approaches may inform theory and improve our nosological understanding of how these constructs interact. Research into factors contributing to psychotherapy response, such as dismantling of multicomponent treatments, is crucial to understanding mechanisms of change. Additionally, more research is needed in non-veteran populations using prospective sleep measures in order to replicate and generalize the findings described above. Further exploration of factors related to risk or resiliency, such as sleep and circadian disruption, following trauma exposure would improve the prospect of early detection and prevention. Given the different dimensions of sleep problems that arise in conjunction with trauma exposure, a holistic perspective of sleep disruption within the context of arousal and negative valence is likely to best inform the next phase of research in this area.

\section{Compliance with Ethical Standards}

Conflict of Interest Monica R. Kelly, William D.S. Killgore, and Patricia L. Haynes declare that they have no conflict of interest.

Human and Animal Rights and Informed Consent This article does not contain any studies with human or animal subjects performed by any of the authors.

\section{References}

Papers of particular interest, published recently, have been highlighted as:

- Of importance

•• Of major importance

1. American Psychiatric Association. Diagnostic and statistical manual of mental disorders. 5th ed. Arlington: American Psychiatric Publishing; 2013.

2. Ross RJ, Ball WA, Sullivan KA, Caroff SN. Sleep disturbance as the hallmark of posttraumatic stress disorder. Am J Psychiatry. 1989;146:697-707. doi:10.1176/ajp.146.6.697.

3. Babson KA, Feldner MT. Temporal relations between sleep problems and both traumatic event exposure and PTSD: a critical review of the empirical literature. J Anxiety Disord. 2010;24:1-15. doi:10.1016/j.janxdis.2009.08.002.

4. Maher MJ, Rego SA, Asnis GM. Sleep disturbances in patients with post-traumatic stress disorder: epidemiology, impact and approaches to management. CNS Drugs. 2006;20:567-90. doi:10.2165/00023210-200620070-00003.

5. Roszell DK, McFall ME, Malas KL. Frequency of symptoms and concurrent psychiatric disorder in Vietnam veterans with chronic PTSD. Hosp Community Psychiatry. 1991;42:293-6.

6. Sinha SS. Trauma-induced insomnia: a novel model for trauma and sleep research. Sleep Med Rev. 2016;25:74-83. doi:10.1016/j.smrv.2015.01.008.

7. Mysliwiec V, O’Reilly B, Polchinski J, Kwon HP, Germain A, Roth BJ. Trauma associated sleep disorder: a proposed parasomnia encompassing disruptive nocturnal behaviors, nightmares, and REM without atonia in trauma survivors. J Clin Sleep Med. 2014;10:1143-8. doi:10.5664/jcsm.4120.

8. Shalev AY, Ankri Y, Gilad M, Israeli-Shalev Y, Adessky R, Qian M, et al. Long-term outcome of early interventions to prevent posttraumatic stress disorder. J Clin Psychiatry. 2016. doi:10.4088/JCP.15m09932.

9. Sanislow CA, Quinn KJ, Sypher I. NIMH research domain criteria (RDoC). Psychol: Encycl. Clin; 2015.

10. Insel T, Cuthbert B, Garvey M, Heinssen R, Pine DS, Quinn K, et al. Research domain criteria (RDoC): toward a new classification framework for research on mental disorders. Am J Psychiatry. 2010;167:748-51. doi:10.1176/appi.ajp.2010.09091379.

11. Germain A. Sleep disturbances as the hallmark of PTSD: where are we now? Am J Psychiatry. 2013;170:372-82. doi:10.1176/appi.ajp.2012.12040432.

12. Gehrman P, Seelig AD, Jacobson IG, Boyko EJ, Hooper TI, Gackstetter GD, et al. Predeployment sleep duration and insomnia symptoms as risk factors for new-onset mental health disorders following military deployment. Sleep. 2013;36:1009-18. doi:10.5665/sleep. 2798 . 
13. Koffel E, Polusny MA, Arbisi PA, Erbes CR. Pre-deployment daytime and nighttime sleep complaints as predictors of postdeployment PTSD and depression in National Guard troops. J Anxiety Disord. 2013;27:512-9. doi:10.1016/j.janxdis.2013.07.003.

14. Galatzer-Levy IR, Bryant RA. 636,120 ways to have posttraumatic stress disorder. Perspect Psychol Sci. 2013;8:651-62. doi:10.1177/1745691613504115.

15. Ressler KJ, Mercer KB, Bradley B, Jovanovic T, Mahan A, Kerley K, et al. Post-traumatic stress disorder is associated with PACAP and the PAC1 receptor. Nature. 2011;470:492-7. doi:10.1038/nature10396.

16. Richter C, Woods IG, Schier AF. Neuropeptidergic control of sleep and wakefulness. Annu Rev Neurosci. 2014;37:503-31. doi:10.1146/annurev-neuro-062111-150447.

17. Almli LM, Mercer KB, Kerley K, Feng H, Bradley B, Conneely $\mathrm{KN}$, et al. ADCYAP1R1 genotype associates with post-traumatic stress symptoms in highly traumatized African-American females. Am J Med Genet Part B Neuropsychiatr Genet. 2013;162:262-72. doi:10.1002/ajmg.b.32145.

18. Stevens JS, Almli LM, Fani N, Gutman DA, Bradley B, Norrholm $\mathrm{SD}$, et al. PACAP receptor gene polymorphism impacts fear responses in the amygdala and hippocampus. Proc Natl Acad Sci U S A. 2014;111:3158-63. doi:10.1073/pnas.1318954111. This study proposes a potential genetic mechanism for amygdala hyperactivity and lack of amygdala-hippocampus connectivity as it relates to risk for PTSD and sleep/wake disturbance.

19. Rosso IM, Weiner MR, Crowley DJ, Silveri MM, Rauch SL, Jensen JE. Insula and anterior cingulate gaba levels in posttraumatic stress disorder: preliminary findings using magnetic resonance spectroscopy. Depress Anxiety. 2014;31:115-23. doi:10.1002/da.22155.

20. Meyerhoff DJ, Mon A, Metzler T, Neylan TC. Cortical gammaaminobutyric acid and glutamate in posttraumatic stress disorder and their relationships to self-reported sleep quality. Sleep. 2014;37:893-900. doi:10.5665/sleep.3654.

21. Altena E, Vrenken H, Van Der Werf YD, van den Heuvel OA, Van Someren EJW. Reduced orbitofrontal and parietal gray matter in chronic insomnia: a voxel-based morphometric study. Biol Psychiatry. 2010;67:182-5. doi:10.1016/j.biopsych.2009.08.003.

22. Killgore WDS, Schwab ZJ, Kipman M, DelDonno SR, Weber M. Voxel-based morphometric gray matter correlates of daytime sleepiness. Neurosci Lett. 2012;518:10-3. doi:10.1016/j.neulet.2012.04.029.

23. Joo EY, Tae WS, Lee MJ, Kang JW, Park HS, Lee JY, et al. Reduced brain gray matter concentration in patients with obstructive sleep apnea syndrome. Sleep. 2010;33:235-41.

24. Nardo D, Högberg G, Jonsson C, Jacobsson H, Hällström T, Pagani M. Neurobiology of sleep disturbances in PTSD patients and traumatized controls: MRI and SPECT findings. Front Psych. 2015. doi:10.3389/fpsyt.2015.00134.

25. Chao LL, Mohlenhoff BS, Weiner MW, Neylan TC. Associations between subjective sleep quality and brain volume in gulf war veterans. Sleep. 2014;37:445-52. doi:10.5665/sleep.3472.

26. Woon FLFL, Sood S, Hedges DW. Hippocampal volume deficits associated with exposure to psychological trauma and posttraumatic stress disorder in adults: a meta-analysis. Prog Neuropsychopharmacol Biol Psychiatry. 2010;34:1181-8. doi:10.1016/j.pnpbp.2010.06.016.

27. Mohlenhoff BS, Chao LL, Buckley ST, Weiner MW, Neylan TC. Are hippocampal size differences in posttraumatic stress disorder mediated by sleep pathology? Alzheimers Dement. 2014. doi:10.1016/j.jalz.2014.04.016.

28. Rauch SL, Shin LM, Phelps EA. Neurocircuitry models of posttraumatic stress disorder and extinction: human neuroimaging research-past, present, and future. Biol Psychiatry. 2006;60:376-82. doi:10.1016/j.biopsych.2006.06.004.

29. Stevens JS, Jovanovic T, Fani N, Ely TD, Glover EM, Bradley B, et al. Disrupted amygdala-prefrontal functional connectivity in civilian women with posttraumatic stress disorder. J Psychiatr Res. 2013;47:1469-78. doi:10.1016/j.jpsychires.2013.05.031.

30. Killgore WDS, Britton JC, Schwab ZJ, Price LM, Weiner MR, Gold AL, et al. Cortico-limbic responses to masked affective faces across ptsd, panic disorder, and specific phobia. Depress Anxiety. 2014;31:150-9. doi:10.1002/da.22156.

31. Yoo S-S, Gujar N, Hu P, Jolesz FA, Walker MP. The human emotional brain without sleep - a prefrontal amygdala disconnect. Curr Biol. 2007;17:R877-8. doi:10.1016/j.cub.2007.08.007.

32. Killgore WDS. Self-reported sleep correlates with prefrontalamygdala functional connectivity and emotional functioning. Sleep. 2013;36:1597-608. doi:10.5665/sleep.3106.

33. Kahn-Greene ET, Killgore DB, Kamimori GH, Balkin TJ, Killgore WDS. The effects of sleep deprivation on symptoms of psychopathology in healthy adults. Sleep Med. 2007;8: 215-21. doi:10.1016/j.sleep.2006.08.007.

34. Killgore WDS, Kahn-Greene ET, Lipizzi EL, Newman RA, Kamimori GH, Balkin TJ. Sleep deprivation reduces perceived emotional intelligence and constructive thinking skills. Sleep Med. 2008;9:517-26. doi:10.1016/j.sleep.2007.07.003.

35. Kobayashi I, Boarts JM, Delahanty DL. Polysomnographically measured sleep abnormalities in PTSD: a meta-analytic review. Psychophysiology. 2007;44:660-9. doi:10.1111/j.14698986.2007.00537.x.

36. Mellman TA. Psychobiology of sleep disturbances in posttraumatic stress disorder. Ann N Y Acad Sci. 1997;821:142-9. doi:10.1111/j.1749-6632.1997.tb48275.x.

37. Germain A, Buysse DJ, Nofzinger E. Sleep-specific mechanisms underlying posttraumatic stress disorder: integrative review and neurobiological hypotheses. Sleep Med Rev. 2008;12:185-95. doi:10.1016/j.smrv.2007.09.003.

38. Germain A, James J, Insana S, Herringa RJ, Mammen O, Price J, et al. A window into the invisible wound of war: functional neuroimaging of REM sleep in returning combat veterans with PTSD. Psychiatry Res. 2013;211:176-9. doi:10.1016/j.pscychresns.2012.05.007.

39. Rasch B, Born J. About sleep's role in memory. Physiol Rev. 2013;93:681-766. doi:10.1152/physrev.00032.2012.

40. Ebdlahad S, Nofzinger EA, James JA, Buysse DJ, Price JC, Germain A. Comparing neural correlates of REM sleep in posttraumatic stress disorder and depression: a neuroimaging study. Psychiatry Res Neuroimaging. 2013;214:422-8. doi:10.1016/j. pscychresns.2013.09.007. Glucose metabolism patterns may help explain nightmares in PTSD but not MDD.

41. Walker MP, van der Helm E. Overnight therapy? The role of sleep in emotional brain processing. Psychol Bull. 2009;135:731-48. doi:10.1037/a0016570.

42. Mellman TA, Kobayashi I, Lavela J, Wilson B, Hall Brown TS. A relationship between REM sleep measures and the duration of posttraumatic stress disorder in a young adult urban minority population. Sleep. 2014;37:1321-6. doi:10.5665/sleep.3922.

43. Mellman TA, Bustamante V, Fins AI, Pigeon WR, Nolan B. REM sleep and the early development of posttraumatic stress disorder. Am J Psychiatry. 2002;159:1696-701.

44. Hurwitz TD, Mahowald MW, Kuskowski M, Engdahl BE. Polysomnographic sleep is not clinically impaired in Vietnam combat veterans with chronic posttraumatic stress disorder. Biol Psychiatry. 1998;44:1066-73.

45. Cowdin N, Kobayashi I, Mellman TA. Theta frequency activity during rapid eye movement (REM) sleep is greater in people with resilience versus PTSD. Exp Brain Res. 2014;232:1479-85. doi:10.1007/s00221-014-3857-5.

46. Sériès F, Roy N, Marc I. Effects of sleep deprivation and sleep fragmentation on upper airway collapsibility in normal subjects. Am J Respir Crit Care Med. 1994;150:481-5.

47. Krakow B, Melendrez D, Warner TD, Dorin R, Harper R, Hollifield $\mathrm{M}$. To breathe, perchance to sleep: sleep-disordered breathing and 
chronic insomnia among trauma survivors. Sleep Breath. 2002;6: 189-202. doi:10.1055/s-2002-36593.

48. Lettieri CJ, Williams SG, Collen JF. OSA syndrome and posttraumatic stress disorder. Chest. 2016;149:483-90. doi:10.1378/chest.15-0693.

49.• Tamanna S, Parker JD, Lyons J, Ullah MI. The effect of continuous positive air pressure (CPAP) on nightmares in patients with posttraumatic stress disorder (PTSD) and obstructive sleep apnea (OSA). J Clin Sleep Med. 2014;10: 631-6. doi:10.5664/jcsm.3786. This study highlights the prevalence of comorbid OSA with PTSD, the relationship between REM sleep dysfunctions and nightmares related to OSA, and benefits of OSA treatment on PTSD-related nightmares.

50. Ong JC, Gress JL, San Pedro-Salcedo MG, Manber R. Frequency and predictors of obstructive sleep apnea among individuals with major depressive disorder and insomnia. J Psychosom Res. 2009;67:135-41. doi:10.1016/j.jpsychores.2009.03.011.

51. Capaldi VF, Guerrero ML, Killgore WDS. Sleep disruptions among returning combat veterans from Iraq and Afghanistan. Mil Med. 2011;176:879-88. doi:10.7205/MILMED-D-10-00440.

52. Colvonen PJ, Masino T, Drummond SPA, Myers US, Angkaw AC, Norman SB. Obstructive sleep apnea and posttraumatic stress disorder among OEF/OIF/OND veterans. J Clin Sleep Med. 2015;11: 513-8. doi:10.5664/jcsm.4692.

53. Pace-Schott EF, Germain A, Milad MR. Effects of sleep on memory for conditioned fear and fear extinction. Psychol Bull. 2015;141: 835-57. doi:10.1037/bul0000014.

54. Inman DJD, Silver SMS, Doghramji K. Sleep disturbance in posttraumatic stress disorder: a comparison with non-PTSD insomnia. J Trauma Stress. 1990;3:429-37. doi:10.1007/BF00974782.

55. Dow BM, Kelsoe JR, Gillin JC. Sleep and dreams in Vietnam PTSD and depression. Biol Psychiatry. 1996;39:42-50. doi:10.1016/0006-3223(95)00103-4.

56. Woodward SH, Friedman MJ, Bliwise DL. Sleep and depression in combat-related PTSD inpatients. Biol Psychiatry. 1996;39:182-92. doi:10.1016/0006-3223(95)00104-2.

57. Breslau N, Roth T, Burduvali E, Kapke A, Schultz L, Roehrs T. Sleep in lifetime posttraumatic stress disorder: a community-based polysomnographic study-correction. Arch Gen Psychiatry. 2005;62:172. doi:10.1001/archpsyc.62.2.172.

58. Gehrman PR, Harb GC, Cook JM, Barilla H, Ross RJ. Sleep diaries of Vietnam War veterans with chronic PTSD: the relationships among insomnia symptoms, psychosocial stress, and nightmares. Behav Sleep Med. 2015;13:255-64. doi:10.1080/15402002.2014.880344.

59. Hall Brown TS, Akeeb A, Mellman TA. The role of trauma type in the risk for insomnia. J Clin Sleep Med. 2015;11:735-9. doi: $10.5664 / \mathrm{jcsm} .4846$.

60. Wright KM, Britt TW, Bliese PD, Adler AB, Picchioni D, Moore D. Insomnia as predictor versus outcome of PTSD and depression among Iraq combat veterans. J Clin Psychol. 2011;67:1240-58. doi:10.1002/jclp.20845.

61. Pigeon WR, Campbell CE, Possemato K, Ouimette P. Longitudinal relationships of insomnia, nightmares, and PTSD severity in recent combat veterans. J Psychosom Res. 2013;75:546-50. doi:10.1016/j.jpsychores.2013.09.004.

62. Wallace ML, Iyengar S, Bramoweth AD, Frank E, Germain A. Clarifying heterogeneity of daytime and nighttime symptoms of posttraumatic stress in combat veterans with insomnia. Mil Psychol. 2015;27:212-22. doi:10.1037/mil0000077.

63. Neylan TC, Marmar CR, Metzler TJ, Weiss DS, Zatzick DF, Delucchi KL, et al. Sleep disturbances in the Vietnam generation: findings from a nationally representative sample of male Vietnam veterans. Am J Psychiatry. 1998;155:929-33. doi:10.1176/ajp.155.7.929.
64. Aurora RN, Zak RS, Auerbach SH, Casey KR, Chowdhuri S, Karippot A, et al. Best practice guide for the treatment of nightmare disorder in adults. J Clin Sleep Med. 2010;6:389-401.

65. Cook JM, Harb GC, Gehrman PR, Cary MS, Gamble GM, Forbes D, et al. Imagery rehearsal for posttraumatic nightmares: a randomized controlled trial. J Trauma Stress. 2010;23:553-63. doi:10.1002/jts.20569.

66. Krakow B, Hollifield M, Johnston L, Koss M, Schrader R, Warner $\mathrm{TD}$, et al. Imagery rehearsal therapy for chronic nightmares in sexual assault survivors with posttraumatic stress disorder. JAMA. 2001;286:537. doi:10.1001/jama.286.5.537.

67. Seda G, Sanchez-Ortuno MM, Welsh CH, Halbower AC, Edinger JD. Comparative meta-analysis of prazosin and imagery rehearsal therapy for nightmare frequency, sleep quality, and posttraumatic stress. J Clin Sleep Med. 2015;11:11-22. doi:10.5664/jcsm.4354.

68. Schoenfeld FB, Deviva JC, Manber R. Treatment of sleep disturbances in posttraumatic stress disorder: a review. J Rehabil Res Dev. 2012;49:729-52. doi:10.1682/JRRD.2011.09.0164.

69. De Berardis D, Marini S, Serroni N, Iasevoli F, Tomasetti C, de Bartolomeis A, et al. Targeting the noradrenergic system in posttraumatic stress disorder: a systematic review and meta-analysis of prazosin trials. Curr Drug Targets. 2015;16:1094-106. doi:10.2174/1389450116666150506114108.

70. Khachatryan D, Groll D, Booij L, Sepehry AA, Schütz CG. Prazosin for treating sleep disturbances in adults with post traumatic stress disorder: a systematic review and meta-analysis of randomized controlled trials. Gen Hosp Psychiatry. 2015;39:46-52. doi:10.1016/j.genhosppsych.2015.10.007.

71. Raskind MA, Peterson K, Williams T, Hoff DJ, Hart K, Holmes $\mathrm{H}$, et al. A trial of prazosin for combat trauma PTSD with nightmares in active-duty soldiers returned from Iraq and Afghanistan. Am J Psychiatry. 2013;170:1003-10. doi:10.1176/appi.ajp.2013.12081133.

72. Petrakis IL, Desai N, Gueorguieva R, Arias A, O'Brien E, Jane JS, et al. Prazosin for veterans with posttraumatic stress disorder and comorbid alcohol dependence: a clinical trial. Alcohol Clin Exp Res. 2016;40:178-86. doi:10.1111/acer.12926.

73. Raskind MA, Peskind ER, Kanter ED, Petrie EC, Radant A, Thompson CE, et al. Reduction of nightmares and other PTSD symptoms in combat veterans by prazosin: a placebocontrolled study. Am J Psychiatry. 2003;160:371-3. doi:10.1176/appi.ajp.160.2.371.

74. Davis JL, Wright DC. Randomized clinical trial for treatment of chronic nightmares in trauma-exposed adults. J Trauma Stress. 2007;20:123-33. doi:10.1002/jts.

75. Long ME, Hammons ME, Davis JL, Frueh BC, Khan MM, Elhai JD, et al. Imagery rescripting and exposure group treatment of posttraumatic nightmares in Veterans with PTSD. J Anxiety Disord. 2011;25:531-5. doi:10.1016/j.janxdis.2010.12.007.

76. Hansen K, Höfling V, Kröner-Borowik T, Stangier U, Steil R. Efficacy of psychological interventions aiming to reduce chronic nightmares: a meta-analysis. Clin Psychol Rev. 2013;33:146-55. doi:10.1016/j.cpr.2012.10.012.

77. Balliett NE, Davis JL, Miller KE. Efficacy of a brief treatment for nightmares and sleep disturbances for veterans. Psychol Trauma Theory, Res Pract Policy. 2015;7:507-15. doi:10.1037/tra0000055.

78. Talbot LS, Maguen S, Metzler TJ, Schmitz M, McCaslin SE, Richards A, et al. Cognitive behavioral therapy for insomnia in posttraumatic stress disorder: a randomized controlled trial. Sleep. 2014;37:327-41. doi:10.5665/sleep.3408. Cognitive behavioral therapy for insomnia for individuals with PTSD is effective in improving subjective and objective insomnia, nightmares, and general functioning. Gains are maintained at 6 months posttreatment.

79. Ho FY-Y, Chan CS, Tang KN-S. Cognitive-behavioral therapy for sleep disturbances in treating posttraumatic stress disorder 
symptoms: a meta-analysis of randomized controlled trials. Clin Psychol Rev. 2016;43:90-102. doi:10.1016/j.cpr.2015.09.005.

80. Livingston WS, Rusch HL, Nersesian PV, Baxter T, Mysliwiec V, Gill JM. Improved sleep in military personnel is associated with changes in the expression of inflammatory genes and improvement in depression symptoms. Front Psych. 2015;6:59. doi:10.3389/fpsyt.2015.00059.

81. Gill JM, Saligan L, Woods S, Page G. PTSD is associated with an excess of inflammatory immune activities. Perspect Psychiatr Care. 2009;45:262-77. doi:10.1111/j.1744-6163.2009.00229.x.

82. Swanson LM, Favorite TK, Arnedt JT, Horin E, Arnedt JT. A combined group treatment for nightmares and insomnia in combat veterans: a pilot study. J Trauma Stress. 2009;22:639-42. doi: $10.1002 /$ jts.

83. Nappi CM, Drummond SPA, Thorp SR, McQuaid JR. Effectiveness of imagery rehearsal therapy for the treatment of combat-related nightmares in veterans. Behav Ther. 2010;41: 237-44. doi:10.1016/j.beth.2009.03.003.

84. Haynes PL, Kelly M, Warner L, Quan SF, Krakow B, Bootzin RR. Cognitive behavioral social rhythm group therapy for veterans with posttraumatic stress disorder, depression, and sleep disturbance: results from an open trial. J Affect Disord. 2016;192:234-43. doi:10.1016/j.jad.2015.12.012.

85. Ehlers CL, Frank E, Kupfer DJ. Social zeitgebers and biological rhythms. A unified approach to understanding the etiology of depression. Arch Gen Psychiatry. 1988;45:948-52. doi:10.1001/archpsyc.1988.01800340076012.

86. Galovski TE, Harik JM, Blain LM, Elwood L, Gloth C, Fletcher TD. Augmenting cognitive processing therapy to improve sleep impairment in PTSD: a randomized controlled trial. J Consult Clin Psychol. 2016;84:167-77. doi:10.1037/ccp0000059.

87. Watts BV, Schnurr PP, Mayo L, Young-Xu Y, Weeks WB, Friedman MJ. Meta-analysis of the efficacy of treatments for posttraumatic stress disorder. J Clin Psychiatry. 2013. doi:10.4088/JCP.12r08225.
88. Galovski TE, Monson C, Bruce SE, Resick PA. Does cognitivebehavioral therapy for PTSD improve perceived health and sleep impairment? J Trauma Stress. 2009;22:197-204. doi:10.1002/jts.20418.

89. Kelly M, Emert S, Epstein D, Perkins S, Parthasarathy S, Wilcox J, et al. Sleep onset latency as a proxy of insomnia severity in the characterization of veteran's positive PTSD psychotherapy outcomes. Sleep. 2015;38:A336.

90. Lommen MJJ, Grey N, Clark DM, Wild J, Stott R, Ehlers A. Sleep and treatment outcome in posttraumatic stress disorder: results from an effectiveness study. Depress Anxiety. 2015. doi:10.1002/da.22420.

91. Zayfert C, De Viva JC, DeViva JC. Residual insomnia following cognitive behavioral therapy for PTSD. J Trauma Stress. 2004;17: 69-73. doi:10.1023/B:JOTS.0000014679.31799.e7.

92. Gutner CA, Casement MD, Stavitsky Gilbert K, Resick PA. Change in sleep symptoms across cognitive processing therapy and prolonged exposure: a longitudinal perspective. Behav Res Ther. 2013;51:817-22. doi:10.1016/j.brat.2013.09.008.

93. Haynes PL, Epstein D, Parthasarathy S, Emert S, Kelly M, Perkins S, Wilcox J (2016) Cognitive Behavioral Therapy for PostTraumatic Stress Disorder is Associated with Negligible Change in Daily Sleep Diary Variables. Sleep 39:In Press.

94. Raboni MR, Alonso FFD, Tufik S, Suchecki D. Improvement of mood and sleep alterations in posttraumatic stress disorder patients by eye movement desensitization and reprocessing. Front Behav Neurosci. 2014;8:209. doi:10.3389/fnbeh.2014.00209.

95. Krakow B, Melendrez D, Johnston L, Warner TD, Clark JO, Pacheco M, et al. Sleep-disordered breathing, psychiatric distress, and quality of life impairment in sexual assault survivors. J Nerv Ment Dis. 2002;190:442-52. doi:10.1097/00005053-200207000-00004.

96. Mustafa M, Erokwu N, Ebose I, Strohl K. Sleep problems and the risk for sleep disorders in an outpatient veteran population. Sleep Breath. 2005;9:57-63. doi:10.1007/s11325-005-0016-z. 